19 Revue d'histoire du XIXe siècle

Société d'histoire de la révolution de 1848 et des

révolutions du XIXe siècle

19 | 1999

Aspects de la production culturelle au XIXe siècle

\title{
L'ère de la culture-marchandise
}

Dominique Kalifa

URL : http://journals.openedition.org/rh19/152

DOI : 10.4000/rh19.152

ISSN : $1777-5329$

Éditeur

La Société de 1848

Édition imprimée

Date de publication : 1 décembre 1999

Pagination : 7-14

ISSN : 1265-1354

Référence électronique

Dominique Kalifa, "L'ère de la culture-marchandise », Revue d'histoire du XIXe siècle [En ligne],

19 | 1999, mis en ligne le 29 juin 2005, consulté le 19 avril 2019. URL : http://journals.openedition.org/ rh19/152 : DOl : 10.4000/rh19.152

Ce document a été généré automatiquement le 19 avril 2019

Tous droits réservés 


\title{
L'ère de la culture-marchandise
}

\author{
Dominique Kalifa
}

1 "Plutôt que de s'intéresser aux produits culturels offerts, mieux vaut s'occuper des opérations qui en font usage ", recommandait il y a déjà longtemps Michel de Certeau ${ }^{1}$. On sait qu'il fut suivi par les historiens français du culturel qui, Roger Chartier en tête, ont mis l'accent sur la nécessité de "penser les pratiques culturelles en termes d'appropriation différentielle" ${ }^{2}$. Contre l'approche strictement sémantique qu'a longtemps pratiquée l'histoire des idées et contre les interprétations sémiologiques des textes (dans l'acception large du terme ${ }^{3}$ ) qui tentent aujourd'hui une partie des historiens anglo-saxons, l'historiographie française a insisté sur cette notion centrale d'" appropriation " ${ }^{4}$, s'accordant à privilégier la question des usages sociaux, à tenir les objets culturels pour des " créations sociales " et la consommation qu'ils induisent pour une forme de production ${ }^{5}$.

2 Pour essentiel qu'il soit, ce déplacement des objets à leurs objectivations demeure entravé par la pauvreté des sources, peu enclines, notamment au XIXe siècle, à produire ou à enregistrer des informations de ce type. La réception, c'est devenu un truisme de le dire, fait office de point aveugle réfrénant une histoire culturelle pourtant volontiers expansionniste. Des brèches existent bien sûr, qui passent par les représentations littéraires ou iconographiques, la production normative ou l'écriture ordinaire, mais elles peinent généralement à produire des tableaux ou des perspectives d'ensemble. D'autres entrées peuvent être dégagées, qui consistent à serrer au plus près les différentes étapes menant à la consommation. Récemment étudié ${ }^{6}$, le cas de la librairie, ultime médiation entre le livre et sa lecture, offre un bon exemple de ce genre d'approches. L'attention portée aux formes matérielles de la production ainsi qu'à ses rythmes ou à sa périodicité s'inscrit dans une même visée, soucieuse de mieux apprécier les conditions de sa réception.

On sait en effet combien la matérialité même des objets culturels construit leur signification, combien leur forme et leur structure commandent leur usage. C'est, pour reprendre l'expression de Donald McKenzie, la "fonction expressive" du dispositif formel ${ }^{7}$, dont les transformations peuvent modeler les effets de sens et agir sur la consommation. Autant que les formes, les rythmes de production, les rythmes de 
diffusion et les rythmes de circulation qu'ils induisent, pèsent sur les usages sociaux. En témoigne l'importance du quotidien, devenu au XIXe siècle l'un des lieux et des facteurs essentiels de l'évolution culturelle, la matérialisation exemplaire des diverses " accélérations " 8 , sociale, économique, politique, qui saisissent alors un pays pleinement entré dans une civilisation de la périodicité.

C'est sous ce double aspect que les différentes contributions réunies dans ce dossier se proposent d'interroger la production culturelle du XIXe siècle, envisagée ici en extension et comme un dispositif de nature systémique, fait d'emprunts, d'échanges et de circulations entre les différents registres et les différents supports. Délaissant les contenus et les représentations manifestes, elles mettent l'accent sur les formes matérielles proposées au public, leurs rythmes de reproduction ou de diffusion, s'efforçant de pointer ce qui, dans la matérialité ou la dynamique de leur circulation, peut infléchir ou non les modes de consommation, signalant de ce fait les permanences et les discontinuités, les recompositions ou les lignes de fuite dont l'imbrication traduit la complexité des évolutions culturelles du siècle. Par-delà la diversité de leurs objets et de leurs approches se dégagent ainsi de leur lecture trois enseignements principaux.

Le premier concerne ce que l'on peut appeler l'invention du " médiatique " 9 , c'est-à-dire d'un régime d'abord marqué, aux antipodes d'une culture de l'immédiateté, par la fusion de toute création dans son support et la transformation de celui-ci en marchandise, dotée d'une forte visibilité matérielle, de modes industrialisés de reproduction ou de représentation, de circuits élargis de diffusion. En signalant l'extraordinaire changement de paradigme que constitue, dans le domaine littéraire, la substitution du modèle textuel au modèle discursif, de l'objet médiatisé à la parole médiatrice, de la " littérature-livre " à la " littérature-discours ", Alain Vaillant et Éric Térouanne introduisent d'emblée au cœur de ce changement de régime. C'est en livres, c'est-à-dire en unités comptables et marchandes, que doit désormais s'exprimer l'écrivain, soumis aux contraintes d'une matérialité et d'une périodicité imposées de l'extérieur de la sphère littéraire. Et encore le livre n'est-il rien s'il n'est pas bientôt collection. " Tu seras une collection ", lance Lousteau à Lucien de Rubempré ${ }^{10}$. En montrant, dans le sillage de son livre ${ }^{11}$, combien le principe de la collection en vient à incarner toute la modernité des stratégies éditoriales au XIXe siècle, Isabelle Olivero confirme l'importance de cette mutation, où s'expriment à la fois les ambitions culturelles d'un siècle orgueilleux et optimiste ${ }^{12}$ et l'entrée dans une ère des masses qui est aussi, par nécessité, celle de la sérialité, seule opération capable d'ordonner la matière, de lui donner forme et sens. Le phénomène n'est d'ailleurs pas limité à la seule production imprimée. Étudiant le fonctionnement théâtral sous la Monarchie de Juillet, Jean-Claude Yon note combien l'intensification de la demande et l'accélération des rythmes enferment les auteurs dramatiques dans des logiques nouvelles de production que le "système de traités " a pour but d'organiser. L'œuvre culturelle est désormais inséparable de ses formes de diffusion et de périodicité, lesquelles imposent bientôt leurs règles, celles de la critique conquérante, puis leurs acteurs, ceux que Christophe Charle a appelés les " hommes doubles ", ces médiateurs rendus indispensables par la disproportion croissante entre l'offre culturelle et la capacité de réception du public ${ }^{13}$.

6 Qu'on l'appelle industriel, médiatique ou de masse, ce nouveau régime se caractérise surtout par la transformation de la culture en objet marchand, dont la "valeur incertaine " ${ }^{14}$ ne l'empêche nullement d'être progressivement inséré au cœur du dispositif de la production moderne. Ainsi s'articule-t-il étroitement aux avancées de la 
technologie, comme le montre Isabelle Olivero en soulignant l'importance du facteur technique dans la sortie de "l'ancien régime typographique" qu'orchestre en 1838 l'éditeur Charpentier, ou encore Emmanuel Pernoud lorsqu'il évoque les mutations de la charge ou des fonctions traditionnelles de la caricature que provoque la modernisation des procédés d'impression à la fin du siècle. Le phénomène doit également être associé à cette lente, mais continuelle invention d'un consumérisme culturel qu'engendrent l'élévation des niveaux de vie et la transformation des usages sociaux du temps ${ }^{15}$. Il serait en effet réducteur de ne voir dans cette évolution qu'un simple effet, matériel et commercial, du productivisme ambiant; s'y mêlent aussi, comme le montrent Isabelle Olivero à propos de l'édition ou Sophie-Anne Leterrier pour le monde musical, d'authentiques ambitions intellectuelles, pédagogiques, voire démocratiques, sans parler même de cette dimension, trop souvent négligée, de jouissance et de satisfaction personnelle dont il faut bien que la consommation culturelle soit également porteuse. S'y ajoute encore le poids des nouveaux contextes, démographiques, sociaux, économiques, politiques, qui imposent au pays une extraordinaire expérience d'accélération du temps et de décloisonnement de l'espace, qui est autant expérience " du chaos " que du progrès et de la modernité, aux sources d'une civilisation nouvelle du nombre, de la vitesse et de la périodicité ${ }^{16}$. D'où l'importance du texte d'Emmanuel Pernoud, qui montre comment la foule, dont la caricature s'empare à la fin du XIXe siècle, n'est pas un simple motif, mais tout à la fois un décor, un objet et un acteur, dont l'entrelacs traduit bien les ambiguïtés de cette massification culturelle et sociale.

7 La portée de ces renouvellements formels ne doit cependant pas être surestimée. L'exemple des journaux de 1848 étudiés par Anne-Claude Ambroise-Rendu montre que le nombre (la ruée médiatique du printemps 1848), le prix (un sou pour près de la moitié d'entre eux, bien avant l'initiative du Petit journal) et le souci de toucher le plus large public ne suffisent pas à donner corps à un véritable dispositif médiatique et marchand. Les raisons de cet échec ne résident pas seulement dans l'absence de séduction éditoriale - les "petits journaux" de la fin du siècle n'étaient finalement pas beaucoup plus " lisibles " que leurs prédécesseurs de 1848 -, mais surtout dans l'extrême focalisation politique, le prosélytisme du ton et "l'incompréhension de ce que sont les exigences d'une publication populaire ".

8 Le second grand apport de ces contributions réside dans l'analyse qu'elles offrent des différents effets suscités par ces mutations matérielles. À commencer par ceux qui affectent les modalités mêmes de la création culturelle. Ainsi Jean-Claude Yon montre-t-il combien les procédures d'industrialisation de l'écriture littéraire (division du travail, rationalisation, standardisation, etc.), associées jusqu'ici à la production du romanfeuilleton ${ }^{17}$, furent sans doute initiées par les auteurs dramatiques, entrés en solitaires dans la logique des "ateliers" et des modes de création collective imposée par la demande croissante des théâtres. Le phénomène est décisif, en ce qu'il précipite également cette double et paradoxale évolution de la " fonction-auteur ", profondément discréditée et surdimensionnée à la fois. Et l'on sait combien ce processus, parfaitement lisible dans le cas de Scribe, affecte dès lors un nombre croissant d'auteurs (pensons à Dumas par exemple, ou à tous les romanciers "populaires" de la seconde moitié du siècle). La question des " droits " s'articule étroitement à ces transformations. Comme le notent Jean-Claude Yon et Sophie-Anne Leterrier à propos du théâtre et de la musique, c'est vers le milieu du XIXe siècle, et sous l'effet de ces évolutions, que des pratiques comme l'emprunt, le plagiat ou la contrefaçon, autrefois mieux tolérées, sont 
vigoureusement dénoncées, et que s'établissent les premières réglementations en la matière. Mais ces mutations affectent aussi les contenus et les genres, comme le rappelle Alain Vaillant à propos du roman, dont l'hypertrophie est directement liée à l'émergence de la "littérature-livre" avec laquelle il finit par se confondre. Le phénomène est également perceptible dans l'univers artistique. Analysant les mécanismes d'échanges et de va-et-vient qui s'établissent à la fin du siècle entre la peinture et le dessin de presse, l'étude d'Emmanuel Pernoud montre combien ce dernier infléchit les représentations picturales, provoquant des effets de déclassement et de recyclage, mais aboutissant finalement à une " nouvelle économie de l'image qui libéralise les échanges entre majeur et mineur ". On peut également songer à " l'affichisme " qui contribue au même moment à brouiller les repères traditionnels en légitimant peu à peu, hors des circuits académiques, ce qui est de plus en plus perçu comme un art de rue. " Ainsi l'art vraiment démocratique, parlant à l'âme populaire, compris de la foule, captivant le regard, enchaînant l'attention et l'admiration, éveillant les idées esthétiques, moralisant par elles, se substitue progressivement à l'art solennel ", écrit un journaliste à l'extrême fin du siècle ${ }^{18}$.

9 Mais c'est pourtant davantage en sens inverse que se font sentir les effets principaux, précipitant les réactions de défense ou de repli académique, suscitant la diffusion d'un discours de la déploration qui atteste de la résistance des " créateurs ", écrivains, artistes ou dramaturges, face à l'irruption d'un régime culturel perçu comme illégitime. L'argumentaire et les stratégies de disqualification nés sous la Monarchie de Juillet pour dénoncer la "littérature industrielle " et le roman-feuilleton ${ }^{19}$, et bientôt appelés à devenir une véritable vulgate périodiquement réactivée, affectent pratiquement dans les mêmes termes, et peut-être même quelques années plus tôt, la production théâtrale d'un Scribe et de ses collaborateurs. Le phénomène ici lisible est bien celui que Pierre Bourdieu a mis en lumière pour le champ littéraire ${ }^{20}$, mais dont les modalités s'appliquent à l'ensemble du dispositif culturel, assez tôt bouleversé par ce règne de la culturemarchandise et des rythmes qu'elle impose. À cette offensive jugée irrecevable répond donc l'invention d'un pole " légitime ", autonome, arc-bouté sur une pratique restreinte de la production et progressivement refermé sur une acception étroite de ce que doivent être l'art et la culture.

10 En étudiant les voies qu'emprunte la sphère musicale pour édifier ce pôle savant et légitime, Sophie-Anne Leterrier met surtout en avant les logiques de "bifurcation culturelle " ${ }^{21}$ dont un tel phénomène est évidemment porteur. La disjonction accrue entre musique savante et musique populaire, son inscription dans des lieux, des institutions et des contextes différenciés accentuent le caractère de marqueur social dont les pratiques culturelles sont de plus en plus investies. Aux formes mieux " partagées " de l'Ancien Régime et du début du siècle, et que beaucoup dans les rangs saint-simoniens ou même libéraux souhaitaient encourager, succèdent vers le milieu du siècle des compartimentages sociaux plus stricts, qui bouleversent également les conceptions et les usages culturels du " peuple ". Celui-ci cesse d'être le destinataire privilégié de l'Art pour en devenir la figure repoussoir, incarnation du vulgaire, ou se réfugie alors dans une valeur refuge, largement déréalisée et patrimonialisée, une sorte de " cœur populaire " mis au service de nouvelles "sciences" et de nouveaux projets ${ }^{22}$. Dans tous les cas cependant, l'évolution signale combien l'industrialisation de la production et ses différents effets, loin de conduire à un effacement des différentiations et à une uniformisation des modes de réception, inaugurent à l'inverse l'entrée dans une ère de la 
diffraction, de l'éparpillement et de l'atomisation de la scène culturelle en une multitude de " publics " de plus en plus différenciés.

11 Ces textes invitent enfin à une troisième série de réflexions, qui concernent la périodisation ou les découpes du temps que l'attention au culturel est propre à renouveler. La question est essentielle, et ne se réduit pas à l'usage d'un cadre commode, neutre, ou simplement indispensable ${ }^{23}$. Instrument essentiel de l'historien, la périodisation est une opération décisive qui porte en elle une large part de la compréhension du passé, possédant en effet le pouvoir de faire sourdre ou bien d'escamoter les objets, d'ordonner les filiations et les généalogies, de mettre au jour les structures, les séquences ou les systèmes cohérents de représentations. De façon plus ou moins explicite, toutes les études réunies dans ce dossier interrogent les acceptions et découpages ordinaires du siècle, allant parfois jusqu'à questionner la pertinence même d'un "XIXe siècle ", qui fut sans doute pourtant le premier à se penser lui-même selon le principe séculaire ${ }^{24}$. Ainsi Alain Vaillant, Éric Terouanne et Isabelle Olivero sont-ils amenés à déborder vers l'aval les limites d'un étroit XIXe siècle pour rendre compte des évolutions éditoriales et bibliométriques. Souvent difficiles en raison de l'étroit bornage qu'imposent les usages et les institutions, notamment universitaires, ces épanchements sont nécessaires à la compréhension de phénomènes culturels rarement circonscrits par des logiques comptables strictement indexées sur la vie politique. Les convergences sont nettes également pour signaler les très fortes ruptures du premier XIXe siècle, celles des années 1830 principalement. Même si elle insiste à juste titre sur la double généalogie du principe de la "Bibliothèque ", Isabelle Olivero montre tout l'impact de la " révolution Charpentier" en 1838, rupture à la fois technique, commerciale et intellectuelle, qui porte en elle presque toutes les innovations à venir jusqu'à l'invention du livre de poche. Ce " moment " parait également décisif à Alain Vaillant et Éric Térouanne, qui y voient les débuts de la tyrannie du " livre publié ", à Sophie-Anne Leterrier qui perçoit l'émergence de nouvelles sociabilités musicales, à Jean-Claude Yon qui montre comment la production théâtrale est dès lors pleinement engagée dans de nouvelles logiques de production. Si l'on y adjoint l'invention du roman-feuilleton et les innovations du marché de la presse qu'impulsent Girardin et ses suiveurs, on prend toute la mesure de cette profonde rupture culturelle du début de la Monarchie de Juillet. Une sorte de proto-histoire du régime de masse est ici perceptible, limitée cependant à la seule structure de l'offre, l'ampleur des inégalités sociales et le refus de considérer les " classes inférieures " comme l'acteur essentiel de la dynamique culturelle interdisant l'émergence d'un grand marché de la consommation capable d'y répondre. C'est paradoxalement le journal, dont le principe de périodicité marchande est pourtant aux sources d'une large part de ces transformations, qui peine alors à suivre le mouvement. Il est intéressant de remarquer combien le livre innova plus tôt que le journal, combien " l'édition populaire " précéda la " presse populaire " 25. L'étude d'Anne-Claude Ambroise-Rendu montre clairement où demeurent, en 1848, les priorités d'une presse encore incapable ou non désireuse de penser le monde pour le grand nombre. D'où le caractère décisif de la décennie 1860 qui, outre l'émergence de nouveaux modèles de développement et de domination sociale ${ }^{26}$, introduit d'autres principes de représentation, fondés notamment sur l'écriture du fait divers et "le courage d'être bête", aux sources de la conception moderne du " médiatique " 27 . Mais les innovations du premier XIXe siècle demeurent essentielles, en ce qu'elles inaugurent le règne de la culture-marchandise. Un nouveau régime culturel s'esquisse et se construit alors, dont le principe et le dispositif perdurent jusqu'au milieu du XXe siècle, lorsque la TSF, puis la télévision et les nouveaux médias imposent un autre 
mode dominant de production/consommation, celui de la linéarité, du flux, du continuum médiatique, adapté aux nouveaux usages sociaux du temps et de la consommation culturelle.

Les six contributions réunies dans ce dossier ne permettent bien sûr pas de prendre la mesure de tous ces phénomènes. Simples coups de sonde qui privilégient sans doute trop l'imprimé, elles laissent dans l'ombre bien des évolutions et des aspects majeurs du siècle, et aspirent donc à être prolongées. On regrette notamment que la science, et de façon plus générale la production académique ou savante, ne soient pas davantage convoquées. Plus grave parce que plus négligée encore par l'historiographie est l'absence d'attention portée aux formes alors renouvelées de l'image. La photographie bien sûr, cette invention majeure du siècle qui modifie autant la relation au " réel " que les usages ou les fonctions de l'art ${ }^{28}$, et que les historiens du XIXe siècle n'ont pas encore suffisamment explorés ${ }^{29}$. Mais la remarque vaut aussi pour des productions comme l'affiche ou la publicité, encore très mal connues, et dont l'immense impact exprime pourtant toute une perception du siècle, assimilé au " puffisme ", au " réclamisme ", à la vulgarité d'un monde de plus en plus visuel, pelliculaire, exempt d'épaisseur et de profondeur ${ }^{30}$. Au vrai, c'est tout un " continent " qui manque à l'appel, qui serait celui de l'œil, du regard, du spectacle ou de l'esthétique de la rue, engagé au XIXe siècle dans une gigantesque mutation qui affecte tout à la fois l'évolution de la " culture sensible ", celle des formes, des usages et des pratiques culturelles, celle des discours qu'ils suscitent. Cette entrée dans une sorte de dynamique triomphante du visuel constitue sans doute l'une des ruptures majeures du XIXe siècle, qu'il conviendrait d'étudier dans la voie ouverte par l'historienne américaine Vanessa Schwartz. Dans un livre important qu'il serait nécessaire de traduire en français ${ }^{31}$, celle-ci a en effet montré combien l'activité frénétique du boulevard, le foisonnement du spectacle de la rue, de la morgue, des panoramas ou du musée Grévin faisaient du Paris de la seconde moitié du XIXe siècle une sorte de laboratoire vivant où s'inventait, dans la multiplicité des regards et de leurs "impressions ", toute la modernité de la culture de masse ${ }^{32}$.

\section{NOTES}

1.. Michel de Certeau, L'invention du quotidien, volume 1. Arts de faire (1980), Paris, Gallimard, Folio Essai, 1990.

2.. Roger Chartier, Culture écrite et société. L'ordre des livres (XIVe-XVIIIe siècle), Paris, Albin Michel, 1996, p. 13.

3.. " Par texte, $\mathrm{j}$ 'entends une construction signifiante de significations potentielles opérant à plusieurs niveaux ", écrit John Fiske, (Reading the Popular, Londres, Unwin Hyman, 1990, p. 43), qui identifie comme tel tout objet mettant en œuvre un système de signes ou un système symbolique ouvert à l'interprétation. Voir aussi, sur cette approche en extension de la notion de texte, Donald F. McKenzie, La bibliographie et la sociologie des textes (1985), Paris, Cercle de la librairie, 1991 pour la traduction française, ainsi que 
Brigitte Le Grignou, " Les périls du texte ", dans Réseaux, nº 80, Les Cultural studies, 1996, pp. 107-124.

4.. " L'appropriation telle que nous l'entendons vise une histoire sociale des usages et des interprétations, rapportés à leurs déterminations fondamentales et inscrits dans les pratiques spécifiques qui les construisent ", écrit Roger Chartier, Culture écrite et société, ouv. cité, p. 214.

5.. Pour un bilan récent des ces travaux, appliqués à l'histoire et à la sociologie de la lecture, voir Gérard Mauger, " Ecrits, lecteurs, lectures ", dans Genèses. Sciences sociales et histoire, $n^{\circ} 34,1999$, pp. 144-161.

6.. Jean-Yves Mollier [dir.], Le commerce de la librairie en France au XIXe siècle (1789-1914), Paris, IMEC/MSH éditions, 1997.

7.. Donald F. McKenzie, La bibliographie et la sociologie des textes, ouv. cité.

8. Christophe Studeny, L'invention de la vitesse. France, XVIIIe-XXe siècle, Paris, Gallimard, 1995.

9.. Sur la notion de culture " médiatique ", voir John G. Calwetti, Adventure, Mystery and Romance. Formula Stories as Art and Popular Culture, Chicago/Londres, University of Chicago Press, 1976.

10.. Balzac, Illusions perdues, cité par Jean-Yves Mollier, " Bibliothèques de Babel : collections, dictionnaires et encyclopédies ", dans Alain Corbin et al., L'invention $\mathrm{du}$ XIXe siècle. Le XIXe siècle par lui-même (littérature, histoire, société), Paris, Klincksieck/Presses de la Sorbonne nouvelle, 1999, p. 331.

11.. Isabelle Olivero, L'invention de la collection. De la diffusion de la littérature et des savoirs à la formation du citoyen au XIXe siècle, Paris, IMEC/MSH éditions, 1999.

12.. Je suis sur ce point les remarques de Jean-Yves Mollier, " Bibliothèques de Babel... ", article cité.

13.. Christophe Charle, Paris Fin de siècle. Culture et politique, Paris, Le Seuil, 1998. Sur l'organisation de ce milieu et les pratiques du " journalisme littéraire " à la fin du siècle, voir Christophe Prochasson, Paris 1900. Essai d'histoire culturelle, Paris, Calmann-Lévy, 1999. 14. Voir, sur cette notion importante, la postface de Bernard Miège, Capitalisme et industries culturelles, Presses universitaires de Grenoble, 1984.

15.. Sur la question de la consommation, voir Rosalind Williams, Dream Worlds. Mass Consumption in Late Nineteenth-Century France, Berkeley, University of California Press, 1982, et surtout Gary Cross, Time and Money. The Making of Consumer Culture, Londres/New York, Routledge, 1993. Sur celle des usages du temps, voir Alain Corbin [dir.], L'avènement des loisirs, Paris, Flammarion, 1995.

16. Stephen Kern, The Culture of Space and Time, 1880-1918, Cambridge, Harvard University Press, 1983. Voir aussi Christophe Studeny, L'Invention de la vitesse, ouv. cité.

17.. Lise Queffelec-Dumasy, Naissance du roman populaire moderne à l'époque romantique, thèse de lettres, université Paris IV, 1983 ; Anne-Marie Thiesse, Le roman du quotidien. Lecteurs et lectures populaires à la Belle époque, Paris, Le Chemin vert, 1984.

18.. J. Roux, " L'art de l'affiche et ses derniers chefs-d'œuvre ", dans Revue des revues, 1er janvier 1899, cité par Christophe Prochasson, Paris 1900, ouv. cité, p. 137.

19.. La querelle du roman-feuilleton (1836-1848), textes présentés par Lise Dumasy, Grenoble, Ellug, 1999 (voir le compte rendu qu'en fait Jacques Migozzi dans cette livraison). Sur la récurrence de l'argumentaire à l'encontre de la culture populaire, voir Brian Rigsby, Popular Culture in Modern France. A Study of Cultural Discourse, New York, 1991.

20.. Pierre Bourdieu, Les Règles de l'art. Genèse et structure du champ littéraire, Paris, Le Seuil, 1992. 
21.. J'emprunte l'expression à Laurence W. Levine, Highbrow/Lowbrow. The Emergence of Cultural Hierarchy in America, Cambrige, Harvard University Press, 1988, qui a étudié le phénomène dans les États-Unis du milieu du XIXe siècle.

22.. Voir sur ce point Anne-Marie Thiesse, La construction des identités nationales, Europe XVIIIe-XXe siècle, Paris, Le Seuil, 1999.

23.. On ne suivra donc pas ici Alain Vaillant, Jean-Pierre Bertrand et Philippe Régnier ( Histoire de la littérature française du XIXe siècle, Paris, Nathan, 1998, p. 8) lorsqu'ils écrivent que la périodisation " n'est rien d'autre qu'un cadre commode de présentation, ou un catalyseur pour la réflexion historique, qu'il convient d'oublier et de changer dès qu'il a rempli son office ".

24.. L'invention du XIXe siècle, ouv. cité.

25.. Voir l'importance par exemple des initiatives de Louis Hachette (Jean-Yves Mollier, Louis Hachette, le fondateur d'un empire, Paris, Fayard, 1999) ou des petits éditeurs populaires du milieu du siècle (Claude Witkowski, Monographie des éditions populaires. Les publications illustrées à 20 centimes, les romans à 4 sous (1848-1870), Paris, Pauvert, 1981 ; Claude Witkowski, Les éditions populaires, 1848-1870, Paris, GIPPE, 1997 ; Georges-André Vuaroqueaux, L'édition populaire au XIXe siècle. L'exemple de la famille Bry, maîtrise d'histoire sous la direction de Jean-Yves Mollier, université Paris-X, 1989).

26. Voir sur ce point Christophe Charle, Histoire sociale de la France au XIXe siècle, Paris, Le Seuil, 1991.

27. Dominique Kalifa, "L'entrée de la France en régime "médiatique" : l'étape des années 1860 ", dans Jacques Migozzi [dir.], Mutations médiatiques, mutations génériques, Presses universitaires de Limoges (sous presse).

28. Je renvoie sur ce point à l'article fondamental de Walter Benjamin, " L'œuvre d'art à l'ère de sa reproductibilité technique " (1936), dans Essais II, Paris, Denoël-Gonthier, 1983. 29.. À l'exception bien sûr du travail majeur, mais isolé, d'André Rouillée, L'empire de la photographie. Photographie et pouvoir bourgeois : 1830-1870, Paris, Le Sycomore, 1982. Voir également la récente livraison de Romantisme $\left(\mathrm{n}^{\circ} 105,1999\right)$ consacrée à " L'Imaginaire photographique ".

30.. Voir les remarques de Philippe Hamon, " Affiches ", dans L'invention du XIXe siècle, ouv. cité, pp. 353-362. Sur la publicité, le seul ouvrage disponible est la synthèse, prématurée mais essentielle, de Marc Martin, Trois siècles de publicité en France, Paris, Odile Jacob, 1992.

31.. Vanessa Schwartz, Spectacular Realities. Early Mass Culture in fin-de-siècle Paris, Berkeley, University of California Press, 1998.

32. Voir dans le même sens les livres de Charle Rearick (Pleasures of the Belle époque. Entertainment and Festivity in turn-of-the-century France, New Heaven, Yale University Press, 1985, et The French in Love and War. Popular Culture in the Era of the two World Wars, New York/Londres, Yale University Press, 1997), et de Michael B. Miller (Sanghai on the Metro. Spies, Intrigue and the French between the Wars, Berkeley, University of California Press, 1994) qui prolongent cette idée des liens privilégiés noués entre Paris, la culture française et le divertissement de masse. 


\section{RÉSUMÉS}

Délaissant l'étude des contenus et des représentations, ce dossier vise à interroger la production culturelle du XIXe siècle sous l'angle de sa forme matérielle, de ses rythmes et de sa périodicité, qui contribuent largement à en définir les usages et la signification. Trois enseignements principaux se dégagent à sa lecture. L'invention précoce d'une culture " médiatique " refermée sur ses supports matériels et leur vocation commerciale ; l'industrialisation de la création et ses effets dans la structuration sociale et professionnelle des modes de production et de consommation ; l'importance enfin des années 1830, qui constituent une sorte de proto-histoire du nouveau régime culturel.

Forsaking the study of contents and representations, this issue aims to examine the cultural production of ninetennth-century by the point of his material shape, of his rythms and of his periodicity, who play a great part in shaping his uses and his meaning. Three main contributions come in light from this papers. The early invention of a "mediatic" culture closed upon his material supports and his business aptitude; the industralization of the creation and his consequences in social and professional structuration of production and consommation; the importance of the $1830^{\prime}$ years, sort of a proto-history of the new cultural system.

\section{INDEX}

Mots-clés : Histoire culturelle 